\title{
Is 6-n-propythiouracil (PROP) taster status related to energy intake and macro- and micronutrient intake in a group of Irish adults and children?
}

\author{
S. O’Brien, E. Feeney, A. Scannell, A. Markey and E. R. Gibney \\ UCD Centre for Food \& Health, School of Agriculture, Food Science and Veterinary Medicine, University College Dublin, \\ Belfield, Dublin 4, Republic of Ireland
}

The ability to taste compounds that contain an $\mathrm{N}-\mathrm{C}=\mathrm{S}$ moiety, such as phenylthiocarbamide (PTC) and PROP, is genetically determined $^{(1)}$. Some individuals are unable to taste compounds with such a thiourea moiety, these individuals are known as non-tasters (NT), and make up approximately $30 \%$ of the general Caucasian population. Medium tasters (MT) and super tasters (ST) account for the remaining $70 \%$ (approximately $40 \%$ and $30 \%$ respectively), depending on their perception of taste. PROP taster status has previously been shown to influence body weight ${ }^{(2)}$ macronutrient selection $^{(3)}$ and fat perception ${ }^{(4)}$.

The present study aimed to investigate whether PROP taster status is related to energy intake and micro- and macronutrient intake in adults and children. The data were collected as part of a pilot study of a larger on-going project examining PROP taster status and its effect on the fruit and vegetable intake of Irish schoolchildren and their parents, as well as their knowledge and attitudes to healthy eating. Twenty-three children and twenty-seven adults from south County Dublin participated in the present study. Energy intakes were assessed from diet histories, PROP taster status was established as previously described ${ }^{(5)}$ and BMI was calculated. Statistical analysis was performed using Pearson's correlation.

\begin{tabular}{|c|c|c|c|c|c|c|c|c|c|c|c|c|c|}
\hline \multirow{3}{*}{ PROP rating.. } & \multicolumn{7}{|c|}{ Adults ( $n$ 27) } & \multicolumn{6}{|c|}{ Children ( $n$ 23) } \\
\hline & \multicolumn{2}{|c|}{$1-2$} & \multicolumn{2}{|c|}{$3-7$} & \multicolumn{2}{|c|}{$8-10$} & \multirow[b]{2}{*}{$P$} & \multirow{2}{*}{$\frac{1-2}{\text { NT }(n 0)}$} & \multicolumn{2}{|c|}{$3-7$} & \multicolumn{2}{|c|}{$8-10$} & \multirow[b]{2}{*}{$P$} \\
\hline & NT $(n$ 3) & $\mathrm{SD}$ & MT (n 13) & SD & ST $(n 11)$ & SD & & & MT $(n$ 11) & SD & ST $\left(\begin{array}{lll}n & 13\end{array}\right)$ & SD & \\
\hline Age (years) & 42.3 & 3.10 & 45.0 & 3.53 & 43.8 & 1.47 & NS & - & 11.9 & 0.29 & 11.8 & 0.40 & NS \\
\hline Height (m) & 1.23 & 9.73 & 1.70 & 0.09 & 1.69 & 0.09 & NS & - & 1.47 & 0.07 & 1.45 & 0.06 & NS \\
\hline Weight (kg) & 73.1 & 15.5 & 77.3 & 15.5 & 80.7 & 14.6 & NS & - & 36.6 & 0.09 & 37.8 & 7.15 & NS \\
\hline $\mathrm{BMI}\left(\mathrm{kg} / \mathrm{m}^{2}\right)$ & 25.7 & 2.30 & 26.6 & 2.92 & 28.0 & 3.72 & NS & - & 19.9 & 2.77 & 18.8 & 2.44 & NS \\
\hline Energy (MJ) & 8.51 & 1.25 & 8.04 & 1.74 & 7.13 & 1.82 & NS & - & 8.28 & 2.14 & 14.8 & 23.4 & NS \\
\hline Carbohydrate & 222 & 28.2 & 212 & 55.9 & 180 & 39.0 & NS & - & 263 & 94.6 & 471 & 746 & NS \\
\hline Fat & 81.1 & 24.3 & 72.8 & 29.2 & 69.3 & 26.6 & NS & - & 74.3 & 18.7 & 132 & 209 & NS \\
\hline Protein & 86.6 & 9.96 & 81.8 & 16.0 & 69.6 & 17.1 & NS & - & 75.5 & 15.5 & 136 & 216 & NS \\
\hline Vitamin D & 1.03 & 0.22 & 2.16 & 0.86 & 2.37 & 1.54 & NS & - & 2.63 & 2.35 & 3.98 & 36.5 & NS \\
\hline Vitamin $\mathrm{B}_{12}$ & 2.46 & 0.58 & 4.00 & 1.37 & 3.12 & 0.92 & $<0.05$ & - & 5.26 & 3.00 & 7.96 & 12.8 & NS \\
\hline
\end{tabular}

No significance was observed in either adults or children for macronutrient intake. However, vitamin $\mathrm{B}_{12}$ differed significantly across PROP taster groups in adults $(P=0.024)$. A similar trend, although not significant $(P=0.098)$, was also observed in children. Although the reasons for this finding are not yet clear, differences in food choice across taster groups may become more obvious in a larger cohort.

1. Yackinous CA \& Guinard JX (2002) Appetite 38, 201-209.

2. Keller KL \& Tepper BJ (2004) Obesity 12, 904-912.

3. Kamphuis MJW \& Westerterp-Plantenga MS (2003) Physiol Behav 79, 167-172.

4. Bartoshuk LM (1979) Science 205, 934-935.

5. Drewnowski A, Henderson SA \& Shore AM (1997) Chem Senses 22, 27-37. 